\title{
Interaction of Water Waves and a Submerged Parabolic Obstacle in the Presence of a Following Uniform/Shear Current Using RANS Model
}

\author{
Yen-Lung Chen, ${ }^{1}$ Jing-Bo Hung, ${ }^{2}$ Shih-Lun Hsu, ${ }^{2}$ Shih-Chun Hsiao, ${ }^{1}$ and Yuan-Chieh Wu ${ }^{3}$ \\ ${ }^{1}$ Department of Hydraulic and Ocean Engineering, National Cheng Kung University, Tainan 701, Taiwan \\ ${ }^{2}$ Tainan Hydraulics Laboratory, National Cheng Kung University, Tainan 701, Taiwan \\ ${ }^{3}$ Institute of Nuclear Energy Research, Atomic Energy Council, Executive Yuan, Taiwan \\ Correspondence should be addressed to Shih-Chun Hsiao; schsiao@mail.ncku.edu.tw
}

Received 27 December 2013; Accepted 28 April 2014; Published 21 May 2014

Academic Editor: Shaoyong Lai

Copyright (C) 2014 Yen-Lung Chen et al. This is an open access article distributed under the Creative Commons Attribution License, which permits unrestricted use, distribution, and reproduction in any medium, provided the original work is properly cited.

\begin{abstract}
This paper simulates regular waves propagating over a submerged parabolic obstacle in the presence of a uniform/shear current using a two-dimensional numerical model, named COBRAS (Cornell Breaking and Structure). The numerical model solves the Reynolds-Averaged Navier-Stokes (RANS) equations and the free surface deformation is tracked using the volume of fluid method (VOF). The capability of the numerical model to simulate regular waves with a uniform or shear current over a constant water depth is first validated with available analytical solutions and experimental data. Comparisons among the experimental data, analytical solutions, and present numerical results show good agreements. Then, regular waves propagating over a submerged parabolic obstacle with a following current are investigated. Detailed discussions including those on the velocity and vorticity fields and the relation between free surface and vorticity are given.
\end{abstract}

\section{Introduction}

Waves and currents often coexist in marine environments. The propagation of waves over a current field is an important topic for coastal, oceanic engineering practitioners and researchers. For traditional problems of coastal and oceanic engineering, it is more near real hydrodynamic problems with the consideration of current effect. Examples include safety of oil platform and design of breakwater. The interactions between waves and currents are very complicated hydrodynamic phenomena that include many uncertain factors, such as wave type, current profile, and the direction between waves and currents. For instance, under wave interaction with a following uniform current, the wave length is lengthened, and the wave height is decreased. In contrast, for an opposing uniform current, the wave length is shortened, and the wave height increases.

Previous studies on wave-current interaction have mainly focused on the geometry of a flat bed. Specifically, Tsao [1] obtained a third-order solution based on the Stokes-type perturbation expansion method for weakly nonlinear waves on a linear shear current. Brevik [2] and Thomas [3, 4] measured the horizontal velocity distribution and surface elevation in wave-current environment. Chen et al. [5] obtained a third-order trajectory solution for water particles in a two-dimensional (2D) wave-current interaction flow under a Lagrangian framework. They conducted an experiment on regular waves with a uniform current to verify the correctness of the solution. Thomas and Klopman [6] proposed a method to classify the strength of wave-current interaction. They used two dimensionless parameters, namely, wave steepness $(\varepsilon=$ $a K)$ and the ratio of fluid speed to celerity $\left(\delta=U_{C} / C\right)$, to classify strength as weak $(\delta \cong O(\varepsilon))$, moderate $(\varepsilon \ll \delta \ll 1)$, or strong $(\varepsilon \ll 1$ and $\delta \cong O(1))$.

The effectiveness of various types of submerged coastal structures and their influences on neighboring surroundings and hydrodynamic phenomena are always an interesting issue. However, to study our understanding of the complicated dynamics associated with wave-structure interaction, it is necessary to simplify the geometry of coastal structure. For 
instance, a submerged rectangular box has been considered as a simplified model for a submerged breakwater. In fact, many research papers have been published on this specific subject (e.g., $[7-10])$.

Zaman et al. [11], Zaman and Togashi [12], and Zaman and Hiraishi [13] theoretically and experimentally studied the free surface evolution and other nonlinear characteristics in a wave-current field over various bottom configurations. Zaman and Togashi [14] reported experimental results on the interaction of regular waves with following and opposing uniform currents over a submerged parabolic obstacle. Zaman et al. [15] stressed the fact that ignoring the separated flow zone will lead to underestimation of the wave height on the lee side. The present study investigates regular waves with a following uniform/shear current over a submerged parabolic obstacle (Table 2). The characteristics of velocity and vorticity fields are discussed, and the relation between free surface and vorticity is given.

\section{Numerical Model}

The COBRAS (Cornell Breaking and Structure) model used here is a 2D numerical model that solves the 2D ReynoldsAveraged Navier-Stokes (RANS) equations for the mean (ensemble-averaged) flow field. The COBRAS model has been widely applied to coastal problem (e.g., [16-21]). For more detailed information on the numerical model, please refer to the study of Lin and Liu [16].

The analytical solution of second-order nonlinear waves interacting with current was derived by Tsao [1]. The corresponding free surface elevation, velocity components, and modified dispersion relation are

$$
\begin{aligned}
\eta(x, t)= & a \cos (K x-\omega t) \\
& +\left[\frac{1}{2 \tanh (K h)}+\frac{3}{4} \frac{\cosh (K h)}{\sinh ^{3}(K h)}\right. \\
& \left.+\frac{1}{4} \frac{\Omega}{C_{r} K}\left(1+\frac{3 g-2 \Omega C_{r}}{g-\Omega C_{r}} \frac{1}{\tanh ^{2}(K h)}\right)\right] \\
& \times K a^{2} \cos 2(K x-\omega t),
\end{aligned}
$$

$$
\begin{aligned}
u(x, y, t)= & D_{1} K a \cosh [K(h+y)] \cos (K x-\omega t) \\
& +2 D_{2} K a^{2} \cosh [2 K(h+y)] \cos [2(K x-\omega t)] \\
& +\beta+U_{\mathrm{fs}}+\Omega y
\end{aligned}
$$

$$
\begin{aligned}
v(x, y, t)= & D_{1} K a \sinh [K(h+y)] \sin (K x-\omega t) \\
& +2 D_{2} K a^{2} \sinh [2 K(h+y)] \sin [2(K x-\omega t)],
\end{aligned}
$$

$$
\begin{gathered}
C_{r}=\frac{\omega}{K}-U_{\mathrm{fs}}, \\
D_{1}=\frac{C_{r}}{\sinh (K h)},
\end{gathered}
$$

$$
\begin{gathered}
D_{2}=\frac{3}{8} \frac{C_{r} K}{\sinh ^{4}(K h)}+\frac{1}{8} \frac{\Omega \cosh (K h)}{\sinh ^{3}(K h)} \frac{3 g-2 \Omega C_{r}}{g-\Omega C_{r}}, \\
\beta=-\frac{a^{2}\left(2 g-\Omega C_{r}\right)}{4 h C_{r}}, \\
\left(\omega-U_{\mathrm{fs}} K\right)^{2}=\left[g K-\Omega\left(\omega-U_{\mathrm{fs}} K\right)\right] \tanh (K h),
\end{gathered}
$$

where $a$ is the wave amplitude, $g$ is gravity, $K$ is the wave number, $\omega$ is the angular frequency, $h$ is the water depth, $U_{\mathrm{fs}}$ is the current velocity at the free surface, and $\Omega$ is constant vorticity.

The reflection of waves from the downstream boundary is common. To prevent it from affecting the accuracy of the numerical results, the radiation boundary condition is applied to the downstream boundary. The equations of the open boundary condition are

$$
\begin{gathered}
\frac{\partial \phi}{\partial t}+C_{0} \frac{\partial \phi}{\partial x}=0, \\
C_{0}=\sqrt{g(\eta+h),}
\end{gathered}
$$

where $\phi$ represents the physical variable associated with waves (e.g., the mean velocities, mean free surface displacement, etc.) and $C_{0}$ is the celerity at the open boundary, which is approximated by (10) for a shallow water wave, and whose value is constant under a fixed water depth. A no-slip condition is used at the solid boundaries (i.e., bottom and obstacle), and a zero-stress condition is applied to the mean free surface for neglecting the air-flow effect.

To avoid unwanted numerical oscillations at the inflow boundary when generating a wave-current system simultaneously, a ramp function is used to eliminate such a potential instability. The ramp function used here, suggested by Wu et al. [22], is a hyperbolic tangent function (see Figure 1). The dependent variable changes with the independent variable gradually in the early stage, reaching the desired value after about four wave periods. In the numerical simulation, (1)-(3) are multiplied by the ramp function, which is

$$
f=\tanh \left(\frac{t}{T}\right)
$$

where $t$ and $T$ are the time and the wave period, respectively.

\section{Numerical Validation}

In order to validate the capability of the model to model wavecurrent interaction, the model results were compared to those reported by Chen et al. [5] for a uniform current with regular waves over a constant water depth under various wave and current conditions. Then, an analytical solution [1] is used to validate the case of a shear current with regular waves.

3.1. Regular Wave with Uniform Current. The wave and current conditions of Chen et al. [5] are listed in Table 1. The numerical wave flume is $10 \mathrm{~m}$ long and $0.9 \mathrm{~m}$ high. A uniform rectangular grid is utilized with $\Delta x=\Delta y=0.002 \mathrm{~m}$. The total simulation time is $30 \mathrm{~s}$. A comparison of the numerical results and the analytical solution of Chen et al. [5] is shown in Figure 2. Very good agreement can be seen for these cases. 


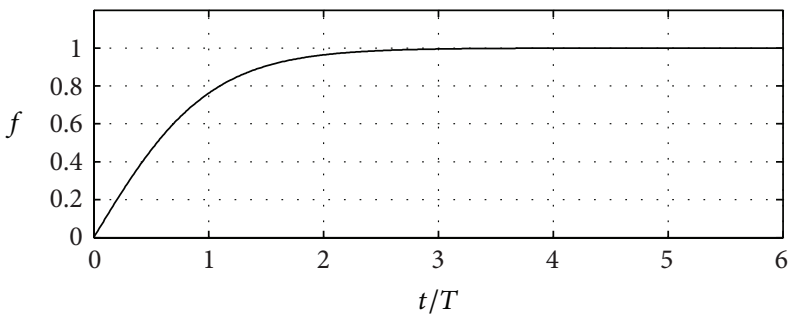

FIgURE 1: Ramp function.
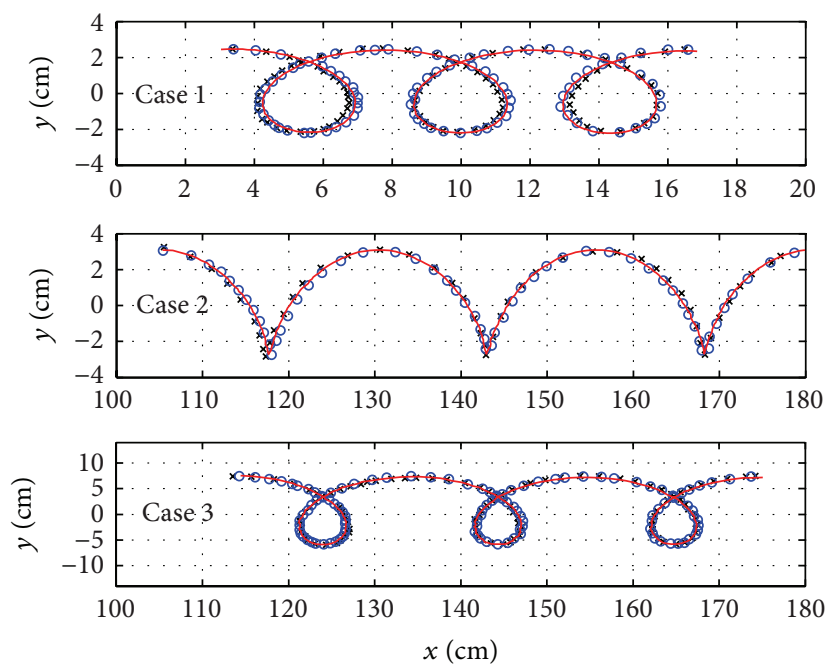

× Lab. (Chen et al., 2012)

- Theory (Chen et al., 2012)

— Present

FIGURE 2: Comparison of particle trajectories for regular waves with a uniform current at constant water depth.

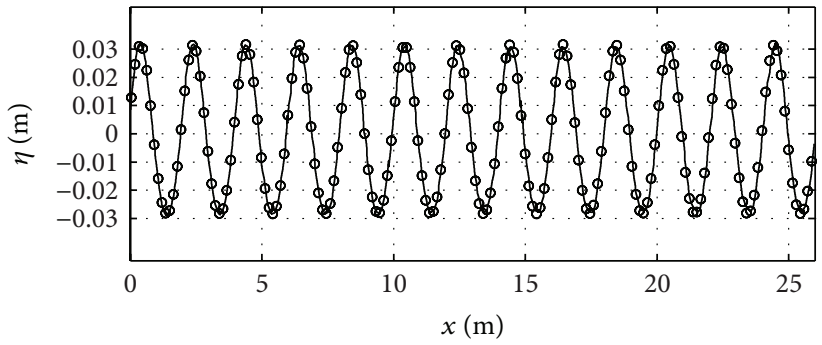

(a)

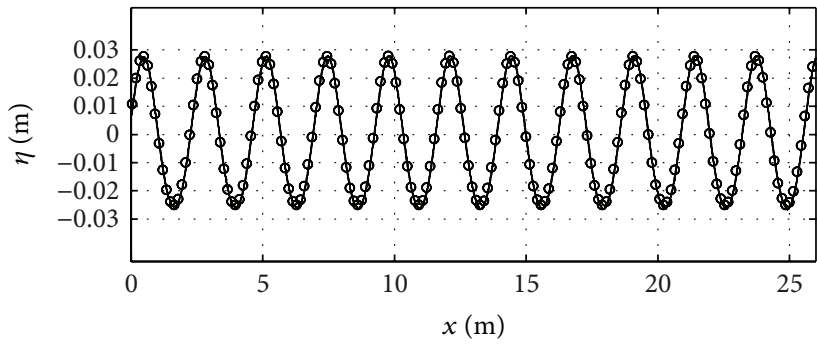

(b)

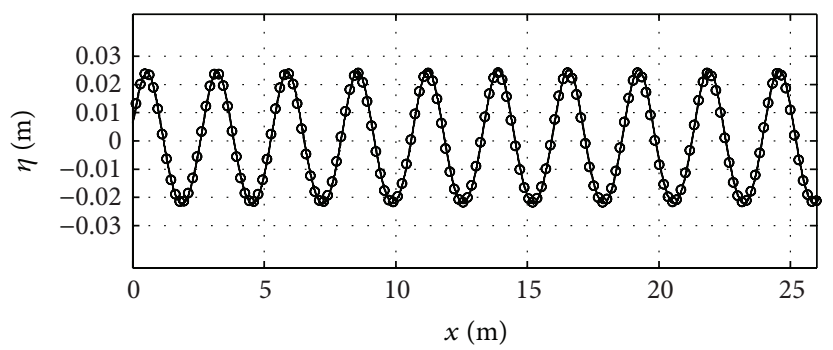

(c)

Figure 3: Spatial surface profiles and comparison of present (-) and Tsao (o). (a) $U=0$, (b) $U=0.24+0.4 y$, and (c) $U=0.54+0.9 y$. 

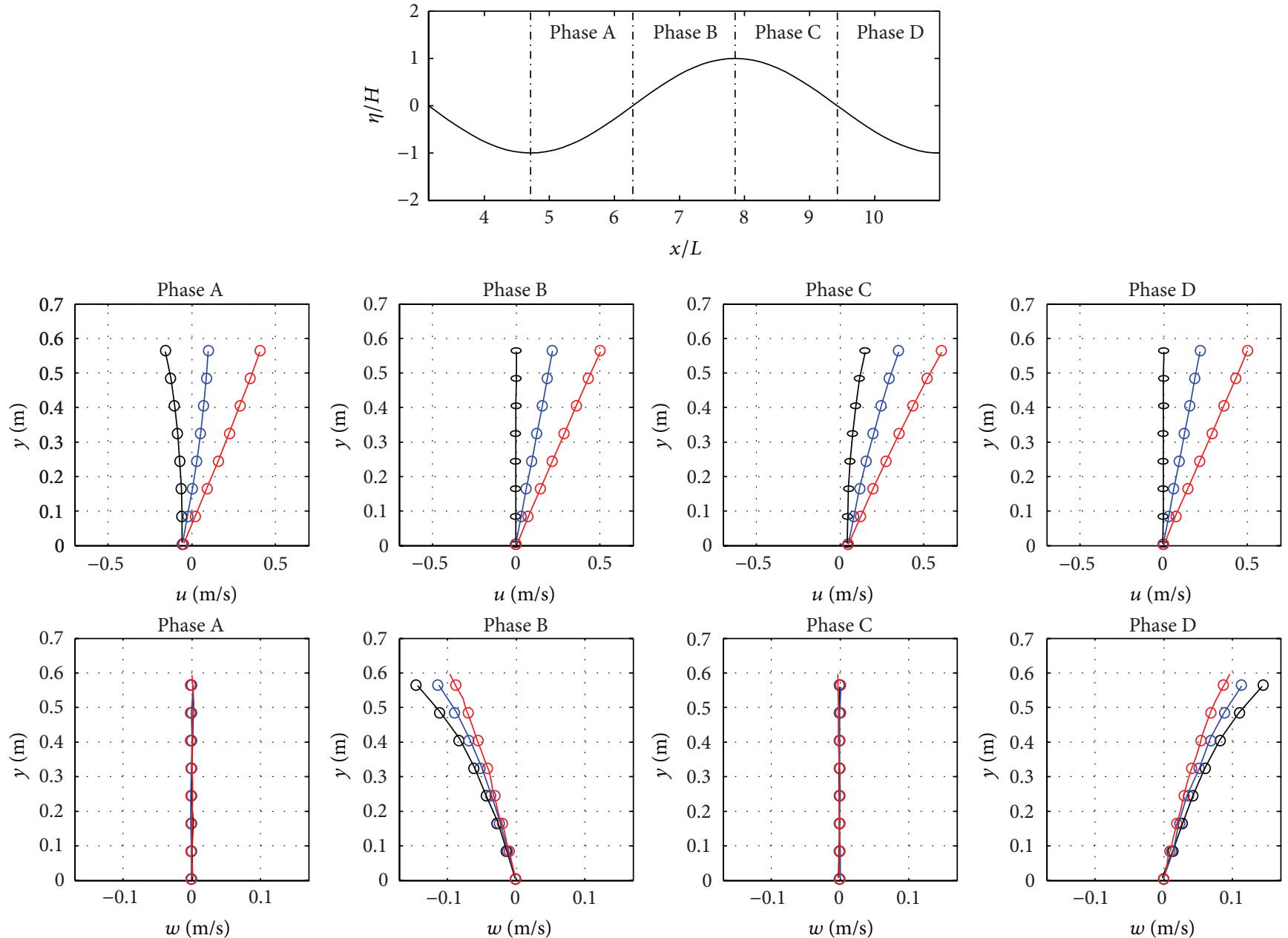

FIGURE 4: Velocity profiles at various phases, comparison of present (-) and Tsao (o), black line is $U=0$, blue line is $U=0.24+0.4 y$, and red line is $U=0.54+0.9 y$.

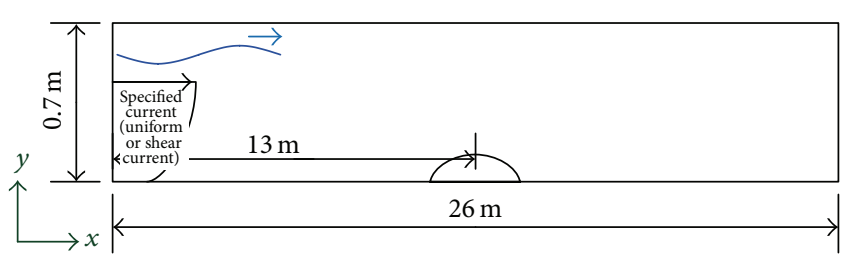

FIGURE 5: Sketch of numerical setup (not to scale).

TABLE 1: Regular waves with uniform current conditions.

\begin{tabular}{lcccc}
\hline & $\begin{array}{c}\text { Water depth } \\
(\mathrm{cm})\end{array}$ & $\begin{array}{c}\text { Water height } \\
(\mathrm{cm})\end{array}$ & $\begin{array}{c}\text { Wave period } \\
(\mathrm{s})\end{array}$ & $\begin{array}{c}\text { Current velocity } \\
(\mathrm{cm} / \mathrm{s})\end{array}$ \\
\hline Case 1 & 50 & 4.62 & 0.99 & 2.95 \\
Case 2 & 80 & 5.82 & 2.06 & 11.72 \\
Case 3 & 80 & 13.20 & 1.00 & 9.63 \\
\hline
\end{tabular}

3.2. Regular Wave with Shear Current. The model results were also compared to the analytical solution given by Tsao [1]. The numerical wave flume is $26 \mathrm{~m}$ long and $0.7 \mathrm{~m}$ high. The water
TABLE 2: Regular waves with shear current conditions.

\begin{tabular}{lcc}
\hline & Current velocity profiles $(\mathrm{m} / \mathrm{s})$ & Strength \\
\hline Case A & 0 & \\
Case B & $0.24+0.4 y$ & Weak \\
Case C & $0.54+0.9 y$ & Moderate \\
\hline
\end{tabular}

depth is $0.6 \mathrm{~m}$, the wave height is $0.06 \mathrm{~m}$, and the wave period is $1.159 \mathrm{~s}$. A uniform rectangular grid is utilized with $\Delta x=$ $\Delta y=0.005 \mathrm{~m}$. The total simulation time is $50 \mathrm{~s}$. Comparisons of the free surface and the velocity are, respectively, shown in Figures 3 and 4 . The spatial free surface elevation and the velocity at various phases well match the analytical solution.

\section{Results and Discussion}

The previous section showed that the proposed model is capable of modeling wave-current interaction. In this section, periodic waves over a parabolic obstacle with a weak/moderate uniform/shear current are simulated. For the geometric setup, please refer to Zaman and Togashi [14]. 


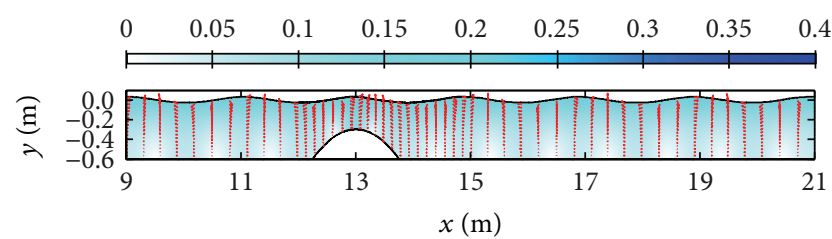

(a)

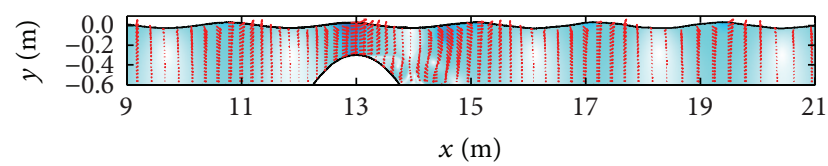

(b)

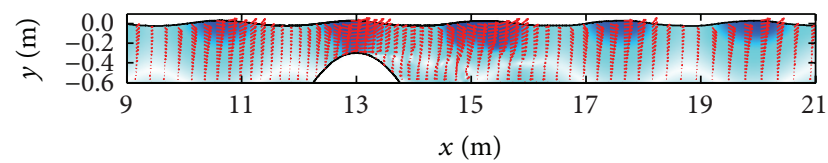

(c)

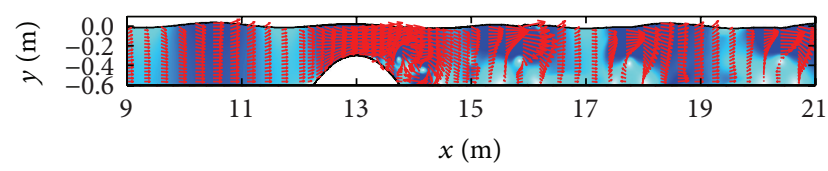

(d)

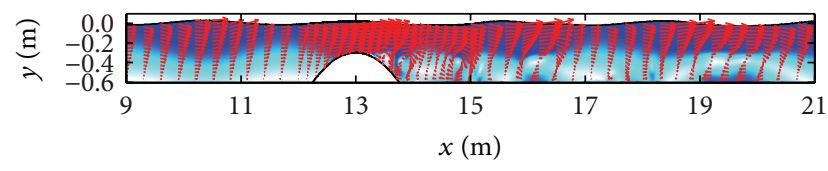

(e)

FIgURE 6: Velocity fields. (a) Pure regular wave and regular waves with a (b) weak uniform current, (c) weak shear current, (d) moderate uniform current, and (e) moderate shear current.

TABLE 3: Mesh allocation.

\begin{tabular}{lccc}
\hline$x(\mathrm{~m})$ & $0 \sim 12$ & $12 \sim 15$ & $15 \sim 26$ \\
$\Delta x(\mathrm{~m})$ & 0.010 & 0.005 & 0.010 \\
$\Delta y(\mathrm{~m})$ & 0.005 & 0.005 & 0.005 \\
\hline
\end{tabular}

Figure 5 shows a schematic diagram of the numerical wave tank, which is $26 \mathrm{~m}$ long and up to $0.7 \mathrm{~m}$ deep. The maximum width and height of the parabolic obstacle are $1.5 \mathrm{~m}$ and $0.3 \mathrm{~m}$, respectively, with the center at $x=13 \mathrm{~m}$. The mesh sizes used in this study are listed in Table 3. The total simulation time is $70 \mathrm{~s}$. Only the data after $40 \mathrm{~s}$ were analyzed because the wave flume was initially unstable. In all cases, the still water depth is $h=0.6 \mathrm{~m}$, and the wave height and wave period of regular waves are $0.06 \mathrm{~m}$ and $1.159 \mathrm{~s}$, respectively. The weak uniform current velocity is $U=0.081 \mathrm{~m} / \mathrm{s}$, and the weak shear current is $U=0.24+0.4 y \mathrm{~m} / \mathrm{s}$; the moderate uniform current velocity is $U=0.297 \mathrm{~m} / \mathrm{s}$, and the moderate shear current is $U=0.54+0.9 y \mathrm{~m} / \mathrm{s}$. The time $t / T=$ 0 was set when the wave crest had just passed the center of the parabolic obstacle. The velocity field, vorticity field, and the relation between the free surface and vorticity were determined.

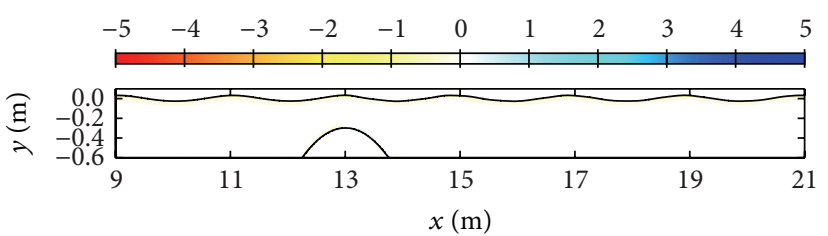

(a)

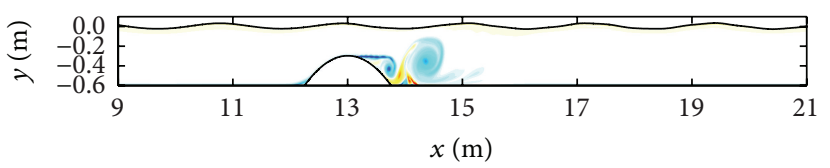

(b)

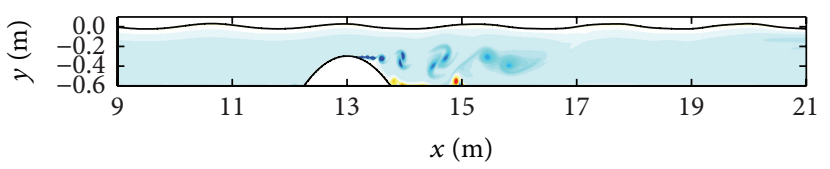

(c)

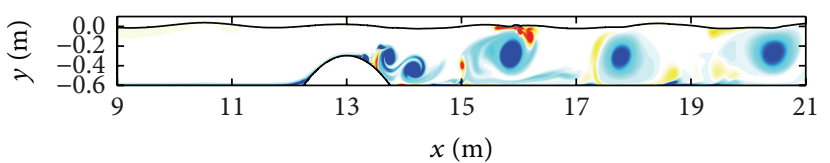

(d)

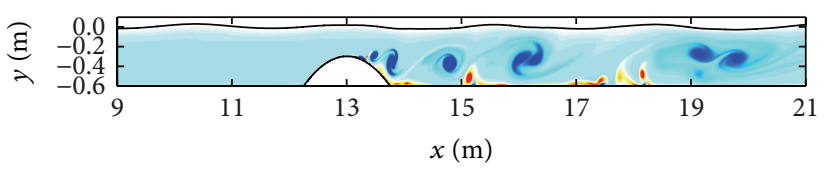

(e)

Figure 7: Vorticity fields. (a) Pure regular wave and regular waves with a (b) weak uniform current, (c) weak shear current, (d) moderate uniform current, and (e) moderate shear current.

The velocity fields are shown in Figure 6. Regular waves with a following current have an increased wave height and a decreased wave length. On the weather side, the velocity field is stable and the velocity distribution is different for uniform and shear currents. The fluid particle velocity in the horizontal direction and the free surface displacement are in phase for regular waves. In contrast, the direction of fluid velocity is always towards the downstream for a following current. Because the horizontal velocity is uniform from the bottom to the free surface for a uniform current and increases linearly from the bottom to the free surface for a shear current, the velocity distribution separates into two layers (i.e., strong and weak regions) in the propagation direction for a uniform current and in the water depth direction for a shear current.

In Figure 6(a), which is the case of regular waves over a submerged parabolic obstacle without any current, the flow field is almost undisturbed. In contrast, the flow field with a current is complicated. In particular, regular waves with a moderate uniform current are the most chaotic (Figure 6(d)). The fluid velocity increases due to the area of flow being decreased by the submerged parabolic obstacle. Because the wave celerity $(C=1.73 \mathrm{~m} / \mathrm{s})$ is faster than the current, the 

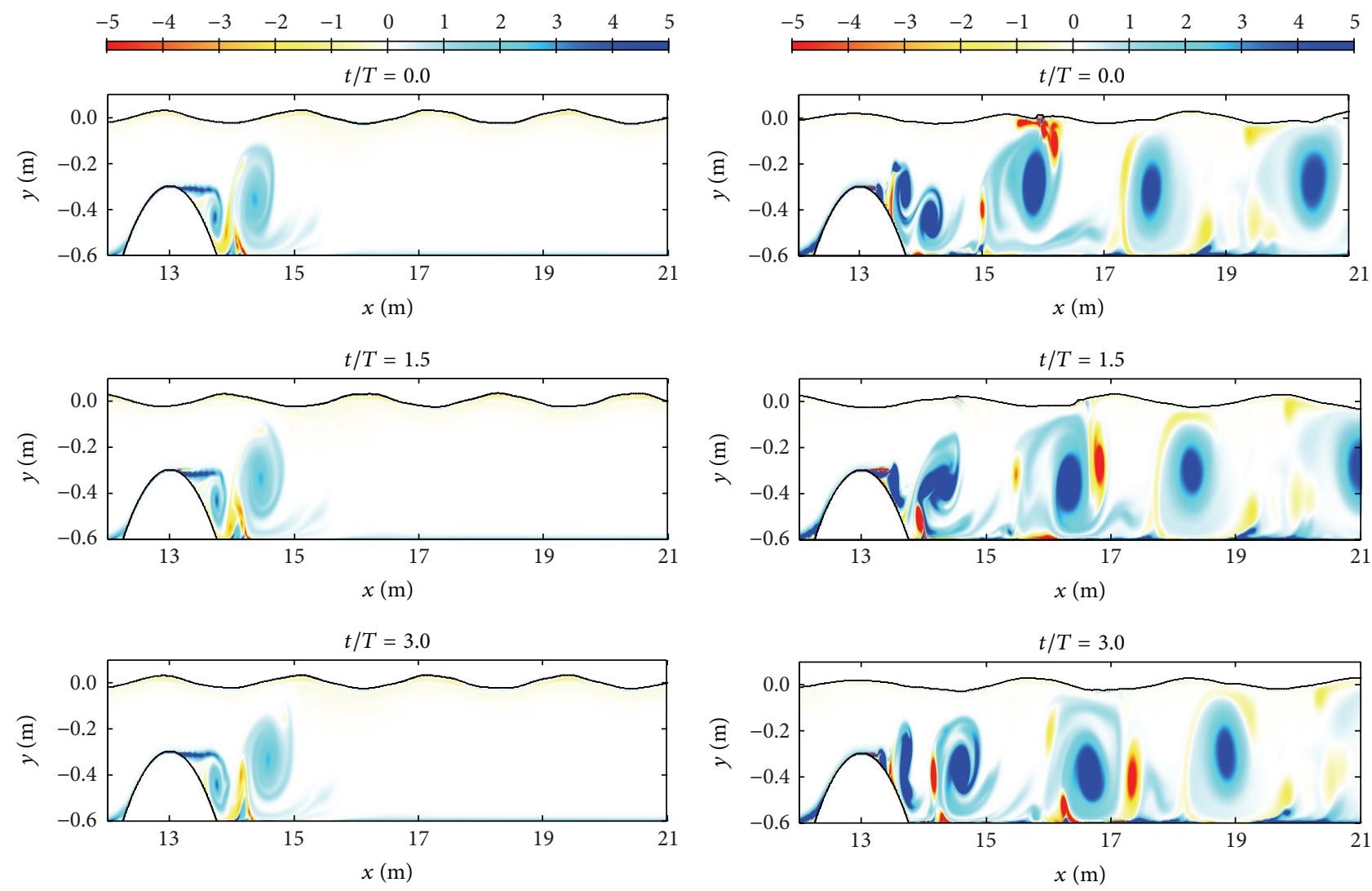

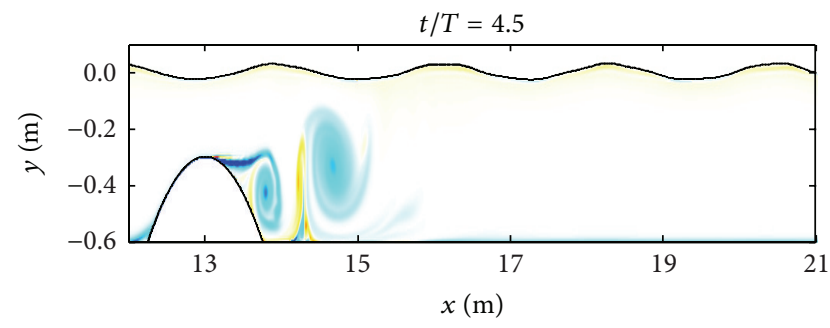

(a)

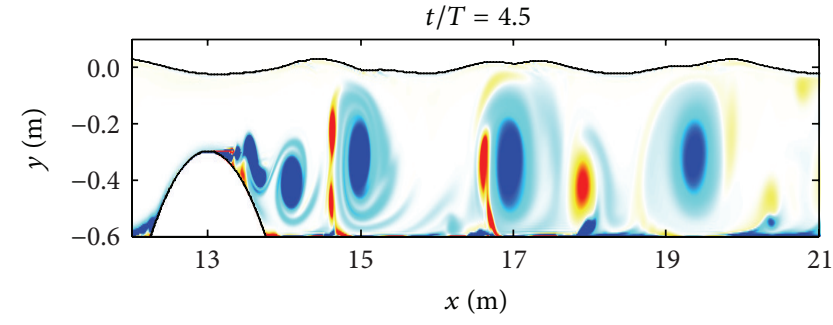

(b)

FIGURE 8: Vortex evolution in the case with uniform current ((a) weak current; (b) moderate current).

increased fluid particles are decreased when they are under the phase of wave trough at the lee side.

The flow over the submerged parabolic obstacle generates vortices from the top of the obstacle or from the sea bottom. No vortex is formed at the lee side of the obstacle as the wave train over the obstacle, as shown in Figure 7(a). In the presence of current, the clockwise vortices originate from the top of the obstacle; the counterclockwise vortices form near the right edge of the obstacle and grow stronger with time (see Figures 7(b)-7(e)). Interestingly, the vortex evolution is like the vortex shedding behind the obstacle. Based on Price et al. [23] and Li et al. [24], the vortex structure only formed from one side can be called the upper shear-layer shedding.

In the presence of a moderate uniform current, if the clockwise vortex is larger than the counterclockwise vortex, the latter will be dropped by the former from the bottom to the free surface, as shown in Figure $7(\mathrm{~d})$. Furthermore, two clockwise vortices mix and form a larger vortex only near the obstacle at the lee side. In the presence of a shear current, the counterclockwise vortex will not be dropped to the free surface since the clockwise vortex is not large enough due to the presence of vorticity in the flow field, as shown in Figures $7(\mathrm{c})$ and $7(\mathrm{e})$. The presence of vorticity in the shear current reduces the scale of the vortices. Therefore, the scale of vortices in a uniform current is larger than that in a shear current.

Figures 8 and 9 show the evolution of vortices with uniform and shear currents, respectively. The vortex evolution is faster and the vortices are larger and stronger when the strength of the current is increased. In Figure 8, the clockwise vortex takes the counterclockwise vortex from the bottom to the free surface, causing free surface disturbance. Interestingly, if the structure of counterclockwise vortex is still complete, it will induce a clockwise vortex from the sea bed. In contrast, the counterclockwise vortices are not 

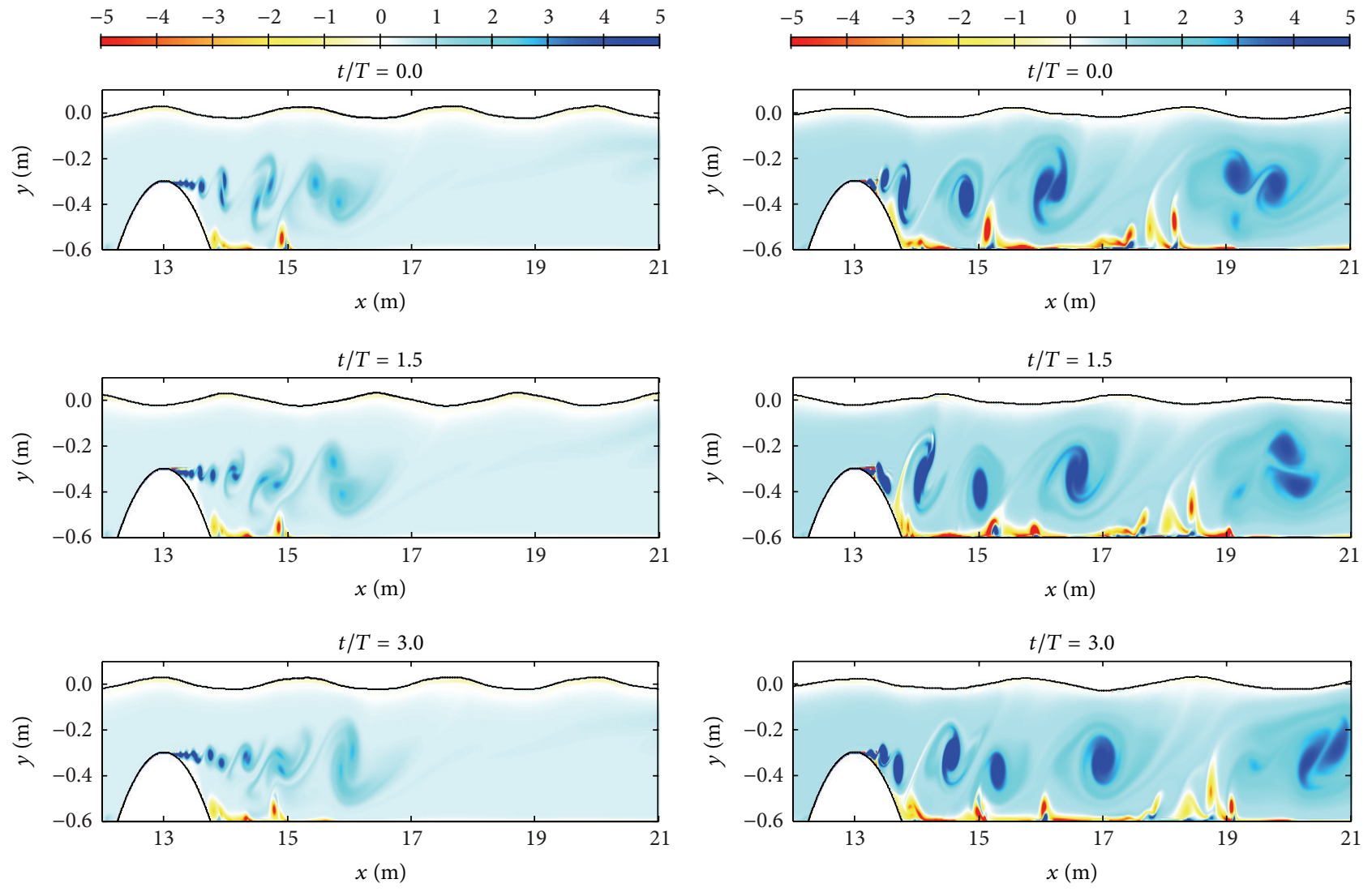

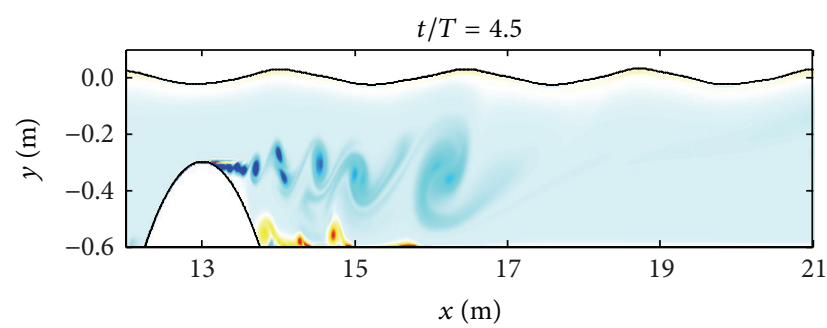

(a)

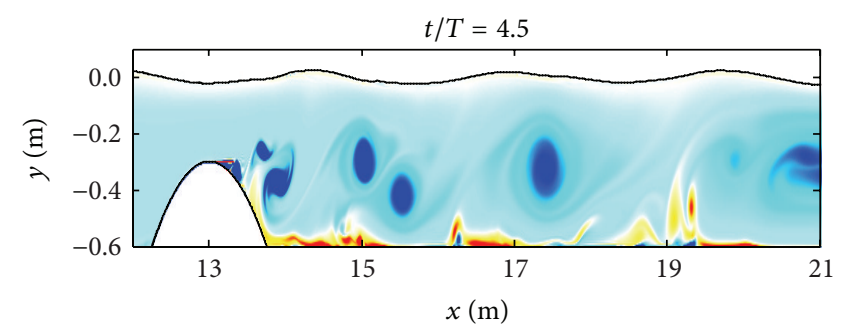

(b)

FIGURE 9: Vortex evolution in the case with shear current ((a) weak current; (b) moderate current).

taken to the free surface for a shear current since the clockwise vortices are small, as shown in Figure 9. At the lee side, the fluid velocity has a nearly linear distribution for a shear current. Therefore, two clockwise vortices at different elevations move at different speeds. Hence, the clockwise vortices will interact and combine, and their strength will decrease due to their interaction. In contrast, the clockwise vortices do not interact in the presence of a uniform current except near the obstacle.

The relation between free surface elevation and vorticity strength at the lee side $(x=16 \mathrm{~m})$ is shown in Figure 10. The vorticity is normalized by the maximum vorticity at the lee side $(x=16 \mathrm{~m})$ for a moderate uniform current. According to Lin and Li [25], the free surface will be sucked down due to the low pressure at the core of vortices. Therefore, the vorticity strength underneath can be estimated by measuring the free surface elevation. The free surface decreases with increasing vorticity strength for a moderate current. In contrast, the free surface elevation is almost independent of vorticity for a weak current. It seems that there exists a threshold value of vorticity above which it affects the free surface elevation.

\section{Conclusions}

The hydrodynamics of regular waves propagating over a submerged parabolic obstacle with the weak/moderate uniform/shear current were investigated. A wave-current function was successfully added to the COBRAS model. The agreement between experimental data, analytical solutions, and the numerical results for wave-current interaction over a flat sea bed is reasonable and consistent with kinematic phenomena $[1,5]$. 


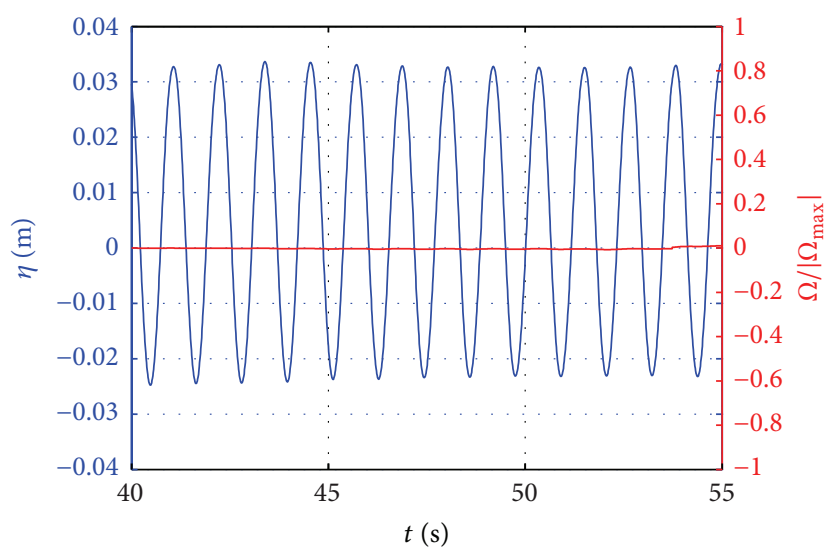

(a)

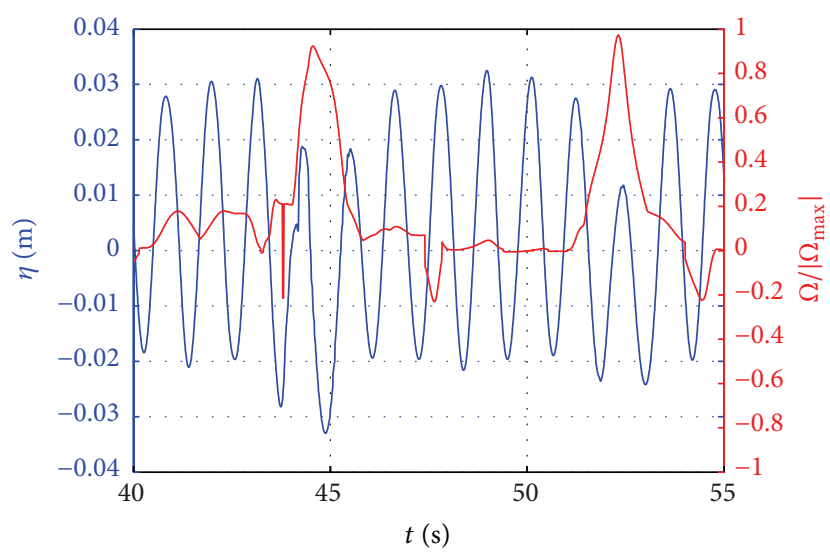

(c)

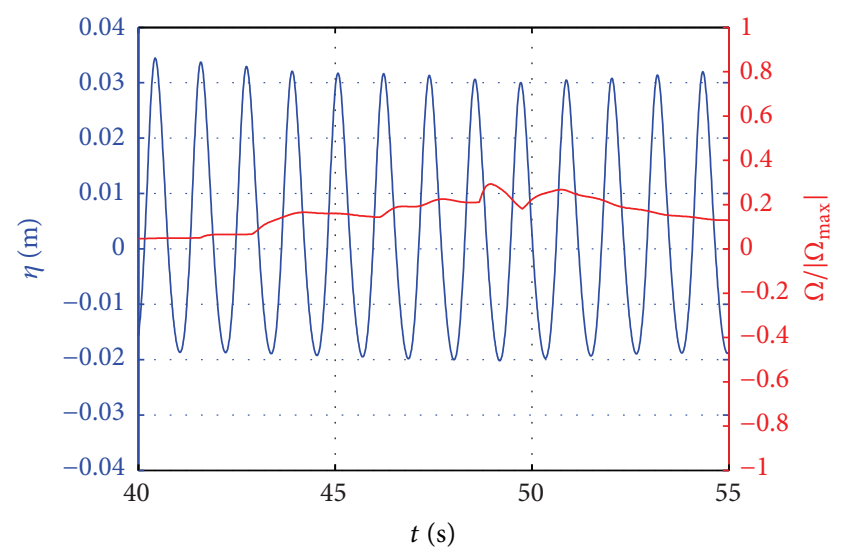

(b)

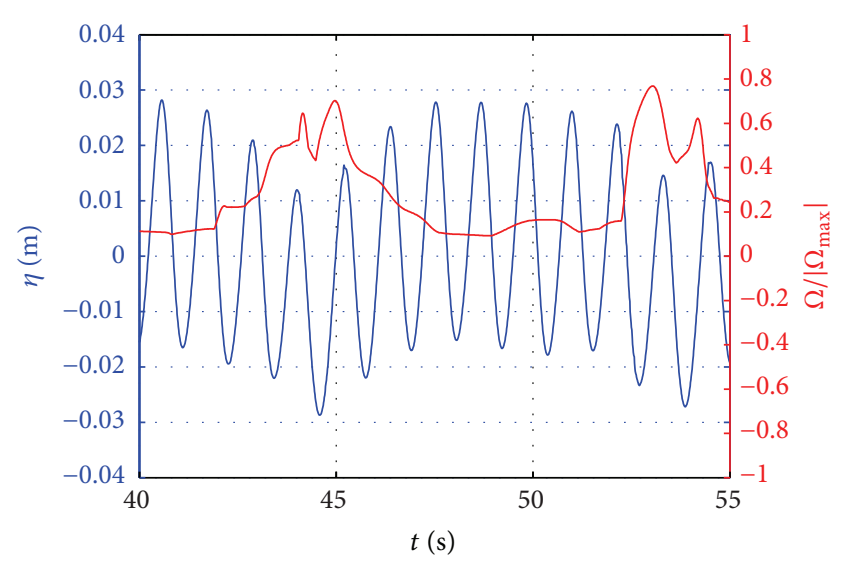

(d)

FIGURE 10: Free surface elevation and vorticity. Regular waves with a (a) weak uniform current, (b) weak shear current, (c) moderate uniform current, and (d) moderate shear current.

From the vorticity field of wave-current propagating a submerged parabolic obstacle, it is found that the presence of vorticity in the flow field (shear current case) leads to a decrease in vortex size. The interaction of clockwise vortices is not occurring in the presence of a uniform current except near the obstacle but occurring in the presence of shear current. Counterclockwise vortices are trapped near the sea bed in the case with a shear current. In contrast, a counterclockwise vortex is dropped to the free surface in the case with a moderate uniform current. Besides, if the structure of counterclockwise vortex is still complete, it will induce a clockwise vortex from the sea bed. For both moderate uniform and shear currents with regular waves over a submerged parabolic obstacle, the free surface decreases with the increasing of vorticity strength.

\section{Conflict of Interests}

The authors declare that there is no conflict of interests regarding the publication of this paper.

\section{Acknowledgments}

This research was financially supported by the National Science Council of Taiwan under Grants NSC 101-2628-E-006015-MY3 and NSC 102-3113-P-006-005 and by the Institute of Nuclear Energy Research, Atomic Energy Council, Executive Yuan, Taiwan, under grants of Project no. 1032001INER043. The authors wish to express their gratitude to Prof. Philip L.-F. Liu, Prof. T.-J. Hsu, and Prof. A. Torres-Freyermuth for their valuable information on the numerical model.

\section{References}

[1] S. Tsao, "Behaviour of surface waves on a linearly varying flow," Moskow. Fiz.Tech. Inst. Issl. Mekh. Prikl. Mat, vol. 3, pp. 66-84, 1959.

[2] I. Brevik, "Flume experiment on waves and currents II. Smooth bed," Coastal Engineering, vol. 4, pp. 89-110, 1980.

[3] G. P. Thomas, "Wave-current interactions: an experimental and numerical study. Part 1. Linear waves," Journal of Fluid Mechanics, vol. 110, pp. 457-474, 1981. 
[4] G. P. Thomas, "Wave-current interactions: an experimental and numerical study. Part 2. Nonlinear waves," Journal of Fluid Mechanics, vol. 216, pp. 505-536, 1990.

[5] Y. Y. Chen, H. C. Hsu, and H. H. Hwung, "Particle trajectories beneath wave-current interaction in a two-dimensional field," Nonlinear Processes in Geophysics, vol. 19, no. 2, pp. 185-197, 2012.

[6] G. P. Thomas and G. Klopman, "Wave-current interactions in the near-shore region," in Gravity Waves in Water of Finite Depth, J. N. Hunt, Ed., Advances in Fluid Mechanics, pp. 255319, Computational Mechanics Publications, 1997.

[7] F. J. Seabra-Santos, D. P. Renouard, and A. M. Temperville, "Numerical and experimental study of the transformation of a solitary wave over a shelf or isolated obstacle," Journal of Fluid Mechanics, vol. 176, pp. 117-134, 1987.

[8] Z. Xie, Theoretical and Numerical Research on Sediment Transport in Pressurised Flow Conditions, University of Nebraska, Lincoln, Mass, USA, 2011.

[9] V. Rey, M. Belzons, and E. Guazzelli, "Propagation of surface gravity waves over a rectangular submerged bar," Journal of Fluid Mechanics, vol. 235, pp. 453-479, 1992.

[10] F. C. K. Ting and Y.-K. Kim, "Vortex generation in water waves propagating over a submerged obstacle," Coastal Engineering, vol. 24, no. 1-2, pp. 23-49, 1994.

[11] M. H. Zaman, H. Togashi, and X. Yu, Modeling Wave Current Interaction over an Uneven Sea Bottom, Civil Engineering in the Ocean, JSCE, Tokyo, Japan, 1994.

[12] M. H. Zaman and H. Togashi, "Modeling horizontally two dimensional wave-current coexistence field over uneven topography," in International Offshore and Polar Engineering Conference (ISOPE '97), 1997.

[13] M. H. Zaman and T. Hiraishi, "Nonlinear model for wave fields with current," Report of the Port and Harbour Research Institute, Ministry of transport, 1999.

[14] M. H. Zaman and H. Togashi, Experimental Study on Interaction Among Waves, Currents and Bottom Topography, Civil Engineering in the Ocean, JSCE, Tokyo, Japan, 1996.

[15] M. H. Zaman, H. Togashi, and R. E. Baddour, "Deformation of monochromatic water wave trains propagating over a submerged obstacle in the presence of uniform currents," Ocean Engineering, vol. 35, no. 8-9, pp. 823-833, 2008.

[16] P. Lin and P. L.-F. Liu, "A numerical study of breaking waves in the surf zone," Journal of Fluid Mechanics, vol. 359, pp. 239-264, 1998.

[17] Y. T. Wu, S. C. Hsiao, Z. C. Huang, and K. S. Hwang, "Propagation of solitary waves over a bottom-mounted barrier," Coastal Engineering, vol. 62, pp. 31-47, 2012.

[18] Y. T. Wu and S. C. Hsiao, "Propagation of solitary waves over a submerged permeable breakwater," Coastal Engineering, vol. 81, pp. 1-18, 2013.

[19] S.-C. Hsiao and T.-C. Lin, "Tsunami-like solitary waves impinging and overtopping an impermeable seawall: experiment and RANS modeling," Coastal Engineering, vol. 57, no. 1, pp. 1-18, 2010.

[20] P. L.-F. Liu and K. Al-Banaa, "Solitary wave runup and force on a vertical barrier," Journal of Fluid Mechanics, no. 505, pp. 225233, 2004.

[21] Y. T. Wu, C. L. Yeh, and S. C. Hsiao, "Three-dimensional numerical simulation on the interaction of solitary waves and porous breakwaters," Coastal Engineering, vol. 85, pp. 12-29, 2014.
[22] C. H. Wu, C.-C. Young, Q. Chen, and P. J. Lynett, "Efficient nonhydrostatic modeling of surface waves from deep to shallow water," Journal of Waterway, Port, Coastal and Ocean Engineering, vol. 136, no. 2, pp. 104-118, 2010.

[23] S. J. Price, D. Sumner, J. G. Smith, K. Leong, and M. P. Païdoussis, "Flow visualization around a circular cylinder near to a plane wall," Journal of Fluids and Structures, vol. 16, no. 2, pp. 175-191, 2002.

[24] W.-J. Li, C. Lin, S.-C. Hsieh, and S. Dey, "Flow characteristics around a circular cylinder placed horizontally above a plane boundary," Journal of Engineering Mechanics, vol. 135, no. 7, pp. 697-716, 2009.

[25] P. Lin and C. W. Li, "Wave-current interaction with a vertical square cylinder," Ocean Engineering, vol. 30, no. 7, pp. 855-876, 2003. 


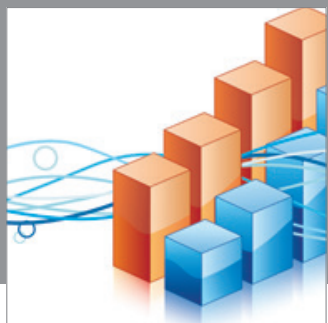

Advances in

Operations Research

mansans

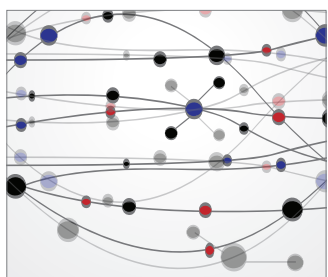

The Scientific World Journal
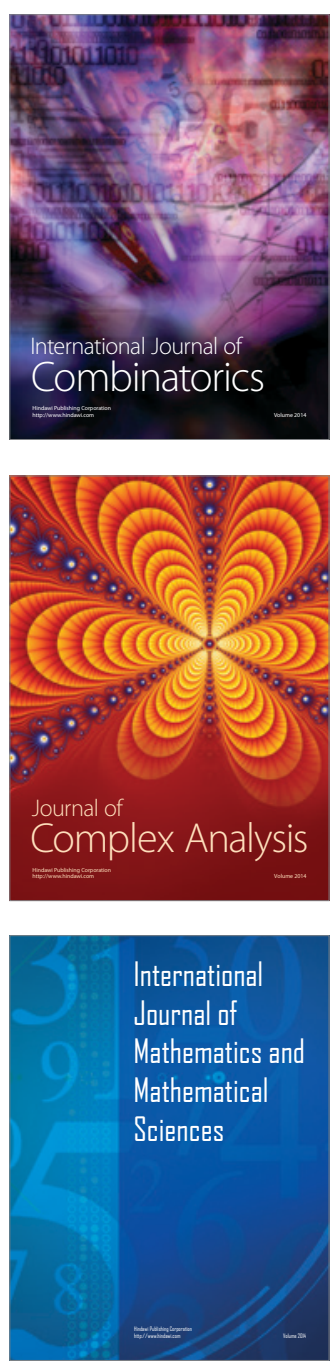
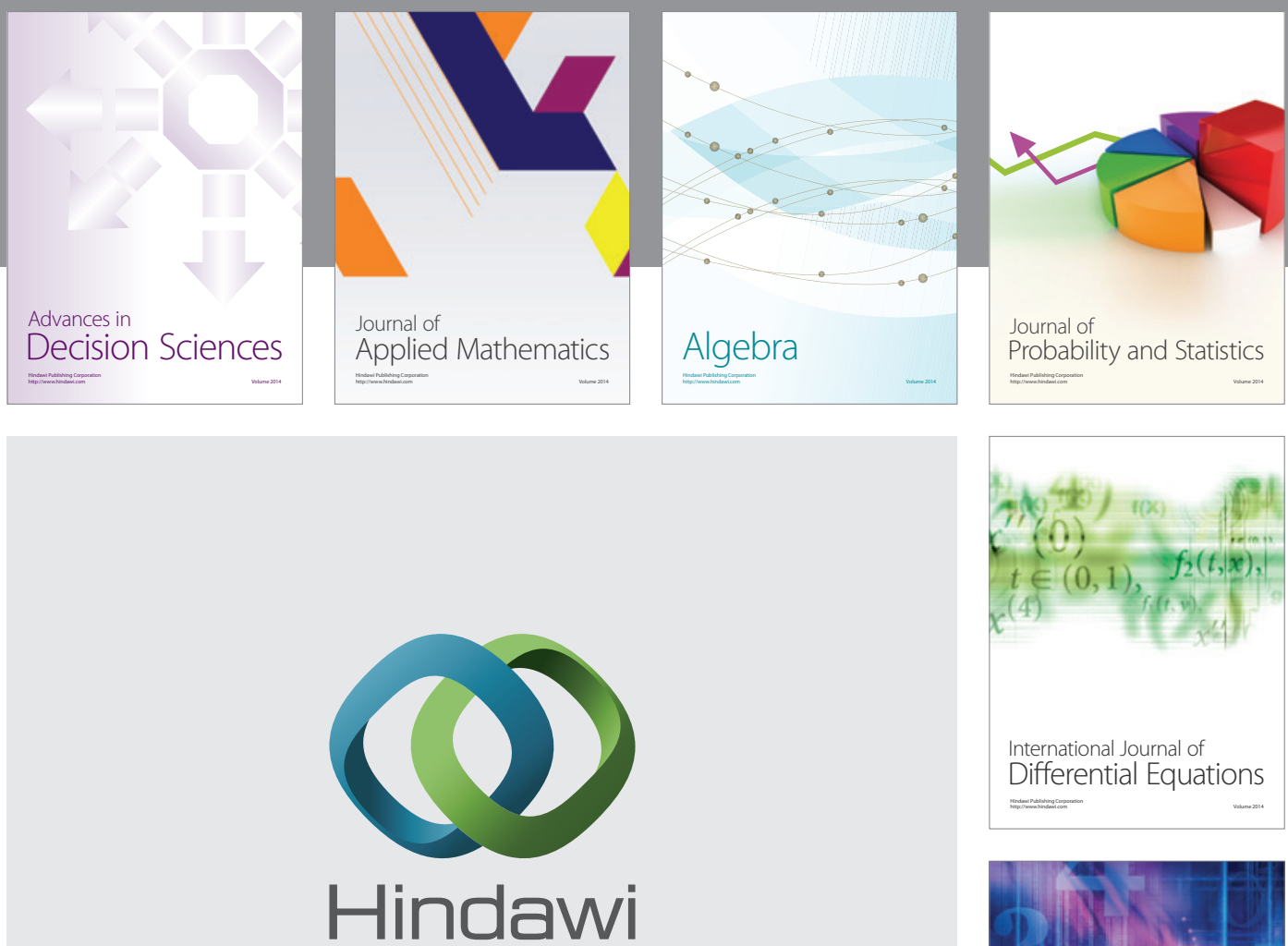

Submit your manuscripts at http://www.hindawi.com
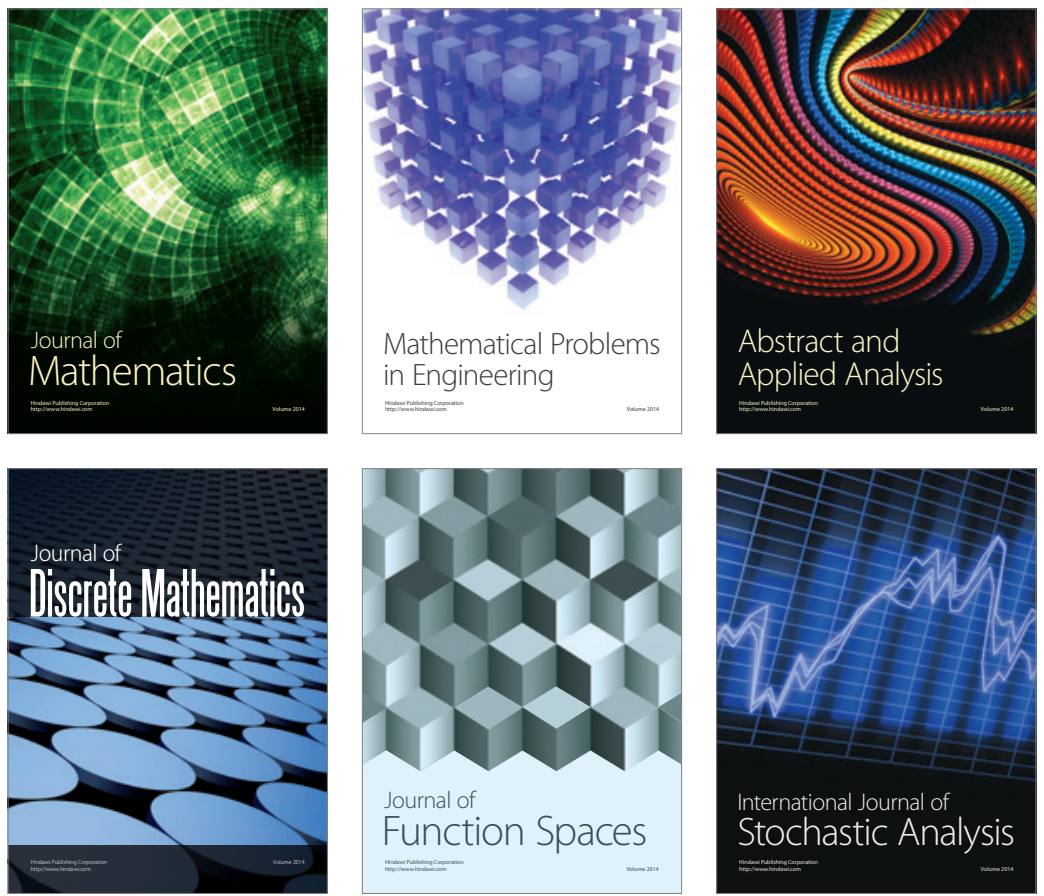

Journal of

Function Spaces

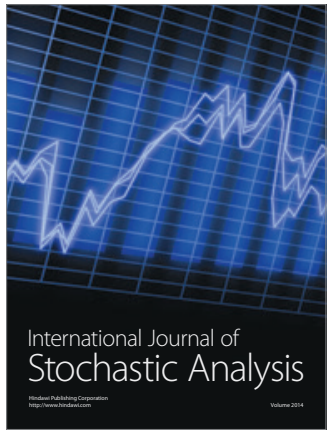

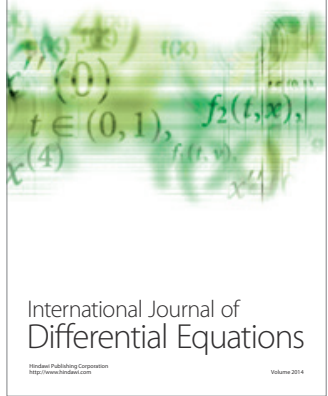
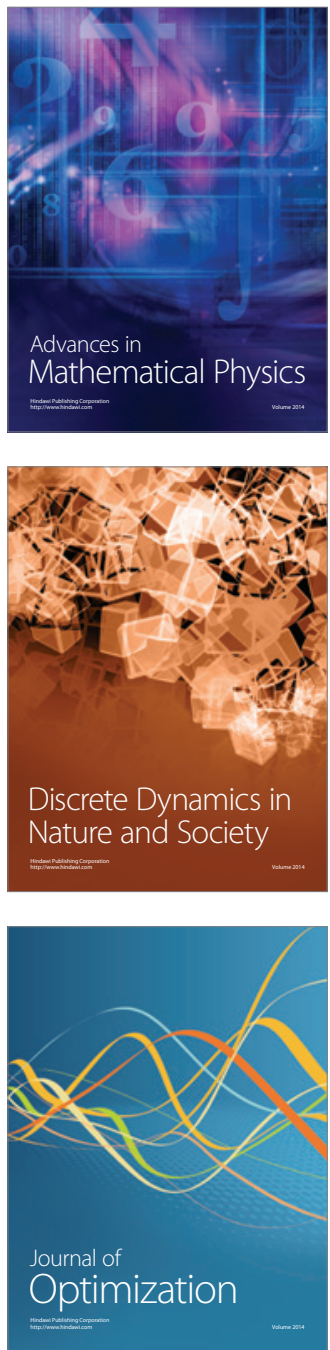